\title{
Forecasting the realized variance of oil-price returns: a disaggregated analysis of the role of uncertainty and geopolitical risk
}

\author{
Rangan Gupta ${ }^{1} \cdot$ Christian Pierdzioch ${ }^{2}$
}

Received: 29 November 2021 / Accepted: 6 February 2022 / Published online: 7 March 2022

(C) The Author(s), under exclusive licence to Springer-Verlag GmbH Germany, part of Springer Nature 2022

\begin{abstract}
We contribute to the empirical literature on the predictability of oil-market volatility by comparing the predictive role of aggregate versus several disaggregated metrics of policy-related and equity-market uncertainties of the USA and geopolitical risks for forecasting the future realized volatility of oil-price (WTI) returns over the monthly period from 1985:01 to 2021:08. Using machine-learning techniques, we find that adding the disaggregated metrics to the array of predictors improves the accuracy of forecasts at intermediate and long forecast horizons, and mainly when we use random forests to estimate our forecasting model.
\end{abstract}

Keywords Realized variance $\cdot$ Oil price $\cdot$ Forecasting $\cdot$ Machine learning $\cdot$ Uncertainty $\cdot$ Geopolitical risk

\section{Introduction}

Historically, movements in the volatility of the returns of the price of crude oil have been found to have predictive value for subsequent slowdowns in worldwide economic growth (van Eyden et al. 2019; Salisu et al. 2021a). At the same time, the recent trend towards financialization that has characterized the development of the oil market, especially during the post the Global Financial Crisis (GFC) era, has implied that the participation of hedge funds, pension funds, and insurance companies in the market has increased. In fact, oil is by now often viewed as an alternative investment in the portfolio decisions of financial institutions (Degiannakis and Filis 2017; Bonato 2019). Naturally, accurate forecasts of the future path of the volatility of oil-price returns are of paramount importance to policymakers in

Responsible Editor: Roula Inglesi-Lotz

Rangan Gupta

rangan.gupta@up.ac.za

Christian Pierdzioch

macroeconmoics@hsu-hh.de

1 Department of Economics, University of Pretoria, Private Bag X20, Hatfield 0028, South Africa

2 Department of Economics, Helmut Schmidt University, Holstenhofweg 85, P.O.B. 700822, 22008 Hamburg, Germany conceptualizing in due time macroeconomic policies to mitigate or even prevent economic recessions, and also for oil traders, because volatility is an important input to investment decisions and portfolio choices (Poon and Granger 2003).

Heightened economic and financial uncertainties, following a series of crises and market jitters such as the GFC, the European sovereign debt crisis, the Brexit, and, of course, the ongoing COVID-19 pandemic, have led researchers to analyze in several significant recent empirical studies the role of uncertainty for forecasting the volatility of the oilprice movements (see, for example, Bonaccolto et al. 2018; Bouri et al. 2020; Li et al. 2020; Liang et al. 2020; Dutta et al. 2021; Gupta and Pierdzioch 2021), which is not surprising given the importance crude oil plays as the most actively traded commodity. In terms of economic theory, the foundation for a positive relationship between uncertainty and the volatility of oil can be derived from the "Theory of Storage," which involves commodity markets in general (Bakas and Triantafyllou 2018, 2020). ${ }^{1}$ According to this theory, increases in uncertainty should make the path of future aggregate demand of commodities, and as a result, also of aggregate production less predictable. Given this

\footnotetext{
${ }^{1}$ In addition, uncertainty may affect the volatility of oil via the so called option-value-to-wait channel (see, e.g., Bernanke 1983). For a recent discussion of the relevance of this channel for the analysis of the volatility of oil, see Van Robays (2016).
} 
heightened unpredictability, risk averse commodity producers prefer to hold physical inventory. In consequence, the convenience yield rises, which, in turn, leads to increased volatility of commodity prices.

In terms of the mushrooming existing literature on uncertainty and its usefulness as a predictor of the future volatility of oil-price returns, Bonaccolto et al. (2018) examine the role of newspapers-based measures of economic-policy and equity-market uncertainty of the United States (U.S.) as predictors of the conditional quantiles of crude-oil returns and volatility by means of a nonparametric $k$-th order causalityin-quantiles framework. They find that these U.S.-based uncertainty indexes are relevant predominantly during periods of market distress, when oil risk is of paramount importance for market participants, where the effects are heterogeneous across different quantiles. Bouri et al. (2020) also use in their empirical analysis daily newspaper-based index of U.S. uncertainty. They, however, focus on uncertainty associated with infectious diseases (EMVID) for oilmarket volatility, and report that adding EMVID to their forecasting models significantly ameliorates forecast accuracy across a range of short-, medium-, and long-run forecast horizons, using a heterogenous autoregressive model of (realized) volatility. A different avenue has been taken by $\mathrm{Li}$ et al. (2020), who apply a so-called mixed-data-sampling generalized autoregressive conditional heteroscedastic (MIDAS-GARCH) model to shed light on the contribution of monetary-policy uncertainty besides overall economicpolicy uncertainty of the U.S. to forecasting oil market volatility. Dutta et al. (2021), however, have shown, based on a quantiles-based modeling framework, that, unlike overall uncertainty of the U.S. related to policy decisions, equitymarket volatility of the U.S. in general, and the same due to commodity market movements and crises, has a higher predictive value for oil-market volatility. In this regard, it is interesting to note that Liang et al. (2020) emphasize the importance of overall equity market volatility indices of the U.S. using a standard predictive regression model, model combination, and shrinkage techniques, while $\mathrm{Li}$ et al. (2020) do not find evidence of a metric of global uncertainty to be important for predicting oil-market volatility. Also taking a global perspective, Gupta and Pierdzioch (2021) compile a dataset for the group of G7 (Canada, France, Germany, Italy, Japan, the United Kingdom (UK), and the U.S.) countries and China to analyze, in an out-of-sample forecasting experiment, the usefulness of of uncertainty and its international spillovers as predictors for the volatility of West Texas Intermediate (WTI) and Brent crude oil-price movements. They use shrinkage estimators for conducting their out-of-sample forecasting experiment and find that uncertainty and international spillovers improve the accuracy of forecasts of the realized volatility of oil returns at intermediate- and long- forecasting horizons.
Against the backdrop of this burgeoning empirical literature on uncertainty and its role for forecasting oil-market volatility, we contribute to this line of research by comparing the role of aggregate versus (ten and forty-four, respectively) disaggregated metrics of policy-related and equity-market uncertainties of the U.S. in predicting the future realized volatility of oil-price (WTI) returns over the monthly period from 1985:01 to 2021:08. Naturally, if disaggregated uncertainty measures, capturing the various multifaceted sources of aggregate policy and equity-market uncertainties, end up producing additional forecasting gains relative to their overall counterparts then this is a finding of considerable value to both investors and policymakers. While it is true, as observed from the concise review presented in the preceding paragraph, that few studies already have studied some of these disaggregated uncertainty measures, we provide a more comprehensive analysis by exhausting all the disaggregated metrics available. In doing so, unlike the existing studies, we use a statistical data-driven approach involving machine-learning methods, rather than randomly deciding on which disaggregate measure to use in the forecasting model of oil-market volatility. In particular, we use a two-step approach. In the first step, we use the least absolute shrinkage and selection operator (Lasso), proposed by Tibshirani (1996). The Lasso belongs to the class of linear regression-analysis methods and performs, in a data-driven way, a regularization of the forecasting model and a selection of predictors so as to improve the interpretability of the model and the accuracy of predictions computed by means of the regularized model. In a second step, we switch to a nonlinear model and estimate random forests (Breiman 2001), which, in turn, is a machine-learning technique tailored to operate in settings featuring a large array of predictors. In particular, random forests automatically capture any potential nonlinear links between the oil market volatility and the (various decomposed) uncertainties as well as any interaction effects between the predictors (for evidence of regime-based nonlinear links between oil-price volatility and macroeconomic uncertainty, see Van Robays (2016); for evidence of interaction effects in the context of a connectedness analysis, see Gabauer and Gupta 2020). In addition to aggregate and disaggregate policy- and equity market-related uncertainties, we also investigate the role of overall geopolitical risks and decomposed values of the same into threats and acts, given that some studies have highlighted the role of such risks too in forecasting oil-market volatility (see for example, Liu et al. 2019; Asai et al. 2020; Mei et al. 2020).

At this stage, it is important to highlight two further points. First, we forecast the monthly realized variance (RV) of returns of the WTI oil price, whereby, following Andersen and Bollerslev (1998), we measure RV in terms of the the sum of daily squared returns over a month, which 
yields an observable and unconditional measure of volatility, which is otherwise a latent process. Conventionally (see the discussions in Chan and Grant 2016 and Lux et al. 2016 for further details), the time-varying volatility is modeled using various models belonging to the GARCH family, under which the conditional variance is a deterministic function of model parameters and past data. Alternatively, some researchers have considered in recent work stochastic volatility (SV) models, which model the volatility as a latent variable that follows a stochastic process. Irrespective of whether one uses GARCH or SV models, the underlying estimate of volatility is not model-free (or unconditional) as in the case of RV. Second, though oil is a globally traded commodity, data on the disaggregated policy and equitymarket uncertainties are only available for the U.S. and, hence, we consider the WTI as our proxy for the global price of oil, with us able to do this given the dominance of the U.S. as a major player in the oil market both on the export and import fronts.

To the best of our knowledge, ours is the first empirical study to compare the role of aggregate and disaggregated measures of policy-, stock-market-, and geopolitical-eventsrelated uncertainties and risks for forecasting the RV of oilprice returns, using linear and nonlinear machine-learning techniques, besides standard Ordinary Least Squares (OLS) applied to the smaller models (in terms of predictors) involving only the aggregate metrics. In the process, our paper adds to the already existing large and significant literature on the predictability of oil-returns volatility by considering the role of various decomposed uncertainties, with the literature having studied the predictive role of a wide array of macroeconomic, financial, behavioral, and climate patterns-related predictors by means of a large spectrum of linear and nonlinear univariate or multivariate models (see, for example, Asai et al. 2019; Bonato et al. 2020; Demirer et al. 2020, 2021; Gkillas et al. 2020; Bouri et al. 2021; Salisu et al. 2021b; Luo Forthcoming, and the references cited within these papers).

We organize the remainder of our paper as follows: Section "Data" contains a description of the data, while "Methods" is devoted to the methodologies. Then, we discuss in "Empirical results" the forecasting results. Section "Concluding remarks" concludes.

\section{Data}

As for the WTI crude oil price, we use the nominal daily data derived from Global Financial Data. ${ }^{2}$ After having computed the daily log-returns (first-differences of the natural logarithm of the WTI oil price), we obtain

\footnotetext{
${ }^{2}$ https://globalfinancialdata.com/.
}

the monthly realized variances, i.e., RV, by summing up the daily squared log-returns over a specific month. Figure 1 plots the resulting realized variance along with its autocorrelation function. Eyeballing the autocorrelation function suggests that a parsimonious autoregressive (AR) model is a useful benchmark model against which we can assess the contribution of EMV, EPU, and GPR and their components to forecast performance.

Turning next to our uncertainty data, it is important to note that uncertainty is a latent variable and, thereby, requires ways to measure it. Gupta et al. (2018) document that three broad approaches have been used in the empirical literature to quantify uncertainty, apart from the various ones associated with financial markets (such as impliedvolatility indices, realized volatility, idiosyncratic volatility of equity returns, corporate spreads). The three approaches can be described as follows: (1) A text-based approach uses the idea is to construct indices of uncertainty from searches of keywords or terms related to (economic and policy) uncertainty in major newspapers or country-reports. (2) A second approach uses stochastic-volatility estimates computed by means of various small- and large-scale structural models (related to macroeconomics and finance) to construct measures of uncertainty. (3) A third approach is to collect data on the dispersion of professional forecaster disagreements to obtain uncertainty estimates. For our metrics of aggregate and disaggregate uncertainties, we use the first approach as outlined by Baker et al. (2016, 2019). For the purpose of our analysis, their approach has several advantages. First, it does not require any complicated estimation of an econometric model to generate it in the first place and, thereby, does not depend on a specific model. Second, their the policy- and equity market-related uncertainty data are available publicly for download. In other words, the aggregate and the corresponding disaggregated measures of uncertainty that we use for our empirical analysis has only been thus far derived from a newspapers-based method, with the ultimate indexes being freely available. In this regard, it is worth emphasizing that the same two advantages also apply in case of the geopolitical risks-related variables that we use as predictors.

The categorical data consists of a range of sub-indexes of the overall economic policy uncertainty (EPU) index, based solely on news data. The sub-indexes are derived using results from the Access World News database of over 2000 U.S. newspapers. Every sub-index requires an article published in these newspapers to contain the terms: "uncertainty" or "uncertain," "economic" or "economy" and one or more of the terms: "congress," "legislation," "white house," "regulation," "federal reserve," or "deficit" as in Baker et al. (2016), as well as a set of categorical policy terms associated with Monetary Policy, Taxes, Fiscal Policy and Government Spending, Health Care, National Security, 
Fig. 1 Realized variance and autocorrelation function
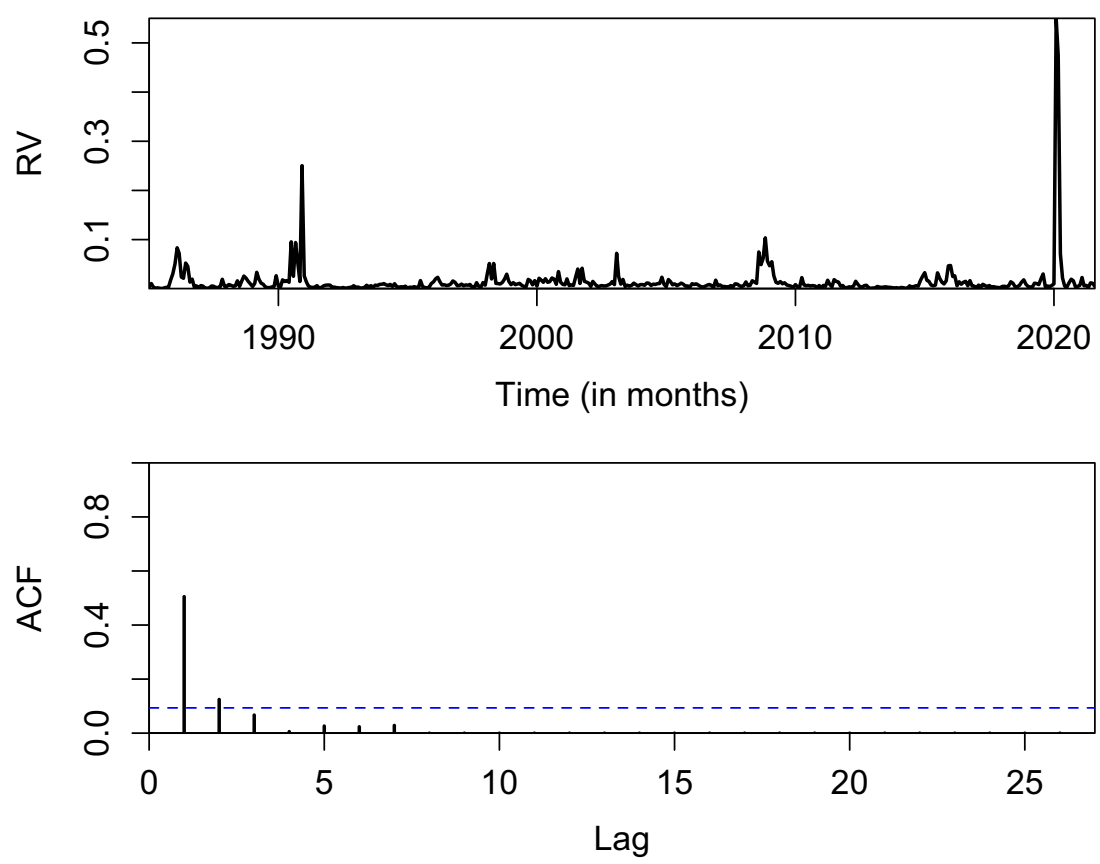

Entitlement Programs, Regulation, Financial Regulation, Trade Policy, and Sovereign Debt and Currency Crises. ${ }^{3}$

Baker et al. (2019) construct a newspaper-based equity market volatility (EMV) tracker that moves with the Volatility Index (VIX) of the Chicago Board Options Exchange (CBOE), and with the realized volatility of returns on the S\&P 500. They further construct an array of 44 category-specific EMV trackers that render it possible to quantify the importance of each category in the level of U.S. stock-market volatility and its movements over time. ${ }^{4}$ In order to compute the overall EMV tracker, Baker et al. (2019) specify terms in the following three sets: E: economic, economy, financial; M: stock market, equity, equities, Standard \& Poors (and variants); and V: volatility, volatile, uncertain, uncertainty, risk, risky. They then obtain monthly counts of newspaper articles (as a percentage of all articles in the same paper and month) that contain at least one term in each of $\mathrm{E}, \mathrm{M}$, and $\mathrm{V}$ from eleven major U.S. newspapers ${ }^{5}$. Finally, Baker et al. (2019) average the scaled counts over newspapers by month, and rescale the

\footnotetext{
${ }^{3}$ The reader is referred to http://policyuncertainty.com/categorical_ terms.html for complete details on the term sets by category of EPU. The overall EPU and the nine categorical sub-indexes are available for download from http://policyuncertainty.com/categorical_epu.html.

${ }^{4}$ The overall and category-specific data are publicly available at http:// policyuncertainty.com/EMV_monthly.html.

${ }^{5}$ The newspapers are the following: The Boston Globe, Chicago Tribune, Dallas Morning News, Houston Chronicle, Los Angeles Times, Miami Herald, New York Times, San Francisco Chronicle, USA Today, Wall Street Journal, and Washington Post.
}

resulting data in a multiplicative way so as to match the mean value of the VIX over the start to the current period of data availability.

In order to obtain the category-specific EMV trackers, Baker et al. (2019) calculate the share of EMV articles in each category and multiply by the contemporaneous EMV tracker value. The EMV tracker categories that they consider are the following: Policy-Related; Infectious Disease; Macroeconomic News and Outlook; MacroBroad Quantity Indicators; Macro-Inflation EMV Indicator; Macro-Interest Rates; Macro-Other Financial Indicators; Macro-Labor Markets; Macro-Real Estate Markets; MacroTrade; Macro-Business Investment and Sentiment; MacroConsumer Spending and Sentiment; Commodity Markets; Financial Crises; Exchange Rates; Healthcare Matters; Litigation Matters; Competition Matters; Labor Disputes; Intellectual Property Matters; Fiscal Policy; Taxes; Government Spending; Deficits, and Debt; Entitlement and Welfare Programs; Monetary Policy Regulation; Financial Regulation; Competition Policy; Intellectual Property Policy; Labor Regulations; Immigration; Energy and Environmental Regulation; Lawsuit and Tort Reform, Supreme Court Decisions; Housing and Land Management; Other Regulation; National Security Policy; Government-Sponsored Enterprises; Trade Policy; Healthcare Policy; Food and Drug Policy; Transportation, Infrastructure, and Public Utilities; Elections and Political Governance; Agricultural Policy; and Petroleum Markets.

Finally, the overall geopolitical risks (GPR), and its subindexes due to threats and attacks are based on the work 
of Caldara and Iacoviello (2019). ${ }^{6}$ The data are derived from an automated text search of the electronic archives of eleven national and international newspapers. ${ }^{7}$ Caldara and Iacoviello (2019) describe that they construct the index by counting the number of articles related to geopolitical risk in each newspaper for each month (as a share of the total number of news articles). The search identifies articles containing references to six groups of words: Group 1 comprises words associated with explicit mentions of geopolitical risk and mentions of military-related tensions involving large regions of the world and a U.S. involvement. Group 2 comprises words directly related to nuclear tensions. Groups 3 and 4 comprise mentions related to war threats and terrorist threats, respectively. Finally, Groups 5 and 6 reflect press coverage of actual adverse geopolitical events (as opposed to risks) which are likely to result in higher geopolitical uncertainty, such as terrorist acts or the beginning of a war. Based on the definitions of the six search groups, Caldara and Iacoviello (2019) further dissect the direct effect of adverse geopolitical events from the effect of pure geopolitical risks. In order to do so, they construct two indexes. The geopolitical threats (GPT) index only includes words belonging to search Groups 1 to 4 . The geopolitical acts (GPA) index only includes words belonging to search Groups 5 and $6 .^{8}$

Figure 2 plots the aggregate EMV, EPU, and GPR data. Based on data availability on $R V$ and the predictors, our analysis covers the monthly period from 1985:01 to 2021:08.

\section{Methods}

An autoregressive (AR) model with one lagged term is our benchmark forecasting model:

$R V_{t+h}=\beta_{0}+\beta_{1} R V_{t}+\eta_{t+h}$,

where $\beta_{i}, i=0,1$ are coefficients to be estimated by the ordinary-least-squares (OLS) technique, and $\eta_{t+h}$ denotes a disturbance term. The parameter, $h$, denotes the forecast horizon. We consider the following forecast horizons: $h=1,3,6,12,24$, where we forecast the average realized variance over the respective forecast horizon for $h>1$. In

\footnotetext{
${ }^{6}$ The data can be downloaded from https://www.matteoiacoviello.com/ gpr2019.htm.

${ }^{7}$ The newspapers are the following: The Boston Globe, Chicago Tribune, The Daily Telegraph, Financial Times, The Globe and Mail, The Guardian, Los Angeles Times, The New York Times, The Times, The Wall Street Journal, and The Washington Post.

${ }^{8}$ The importance of disaggregating the overall GPR index when analyzing its impact on oil market volatility has been highlighted by recent studies (see for example, Demirer et al. 2018, 2019 and Lee et al. 2021).
}

addition, we structure the data matrix so that it has the same dimension for all forecast horizons.

Our first extension of the model given in Eq. 1 features the aggregate EMV, EPU, and GLPR predictors and is given by

$$
\begin{aligned}
R V_{t+h}= & \beta_{0}+\beta_{1} R V_{t}+\beta_{2} E M V_{t}+\beta_{3} E P U_{t} \\
& +\beta_{4} G P R_{t}+\eta_{t+h} .
\end{aligned}
$$

Again, we use the OLS technique to estimate the coefficients of this forecasting model.

Next, we take a disaggregated perspective and use the various categories of EMV, EPU, and GPR (threats and actual geopolitical risks in case of the latter) for forecasting the realized variance. Our forecasting model is of the format

$R V_{t+h}=\beta_{0}+\beta_{1} R V_{t}+\sum_{c=1}^{n} \gamma_{c} x_{t, c}+\eta_{t+h}$,

where $\gamma_{c}$ are the coefficients corresponding to the $n$ categories, $x_{t, c}$. Because of the large number, $n$, of categories, we do not estimate the model given in Eq. 3 by the OLS technique, but rather apply the least absolute shrinkage and selection operator (Lasso), a machine-learning technique proposed by Tibshirani (1996). The Lasso estimates of the model coefficients are chosen to minimize the following expression (for a textbook exposition of the Lasso, see, e.g., Hastie et al. 2009):

$$
\begin{aligned}
& \sum_{t=1}^{N}\left(R V_{t+h}-\beta_{0}+\beta_{1} R V_{t}+\sum_{c=1}^{n} \gamma_{c} x_{t, c}\right)^{2} \\
& +\lambda\left(\left|\beta_{1}\right|+\sum_{c=1}^{n}\left|\gamma_{c}\right|\right)
\end{aligned}
$$

where $N$ is the number of observations available for estimation of the model. Hence, the Lasso estimator is a shrinkage estimator that uses the L1 norm of the vector of coefficients to shrink the dimension of the estimated model. The magnitude of the shrinkage parameter, $\lambda$, governs the extent of the coefficient shrinkage. When $\lambda$ is large enough, the Lasso estimator can even set to zero some of the coefficients, in which case the Lasso estimator can also be interpreted as a predictor-selection estimator.

The forecasting models that we specify in Eqs. 1, 2, and 4 all belong to the class of linear forecasting models. As indicated in the introduction, because there is evidence of the various categories of uncertainty and geopolitical risks being interdependent (see also Caldara and Iacoviello 2019 in this regard), and because the realized variance may be a nonlinear function of these predictors, it is warranted also to consider a nonlinear forecasting model. Given the large number of categories that we consider in our empirical analysis, we, therefore, also consider a forecasting model of the following format:

$R V_{t+h}=f\left(R V_{t}, x_{t, 1}, x_{t, 2}, \ldots x_{t, n}\right)$ 
Fig. 2 EMV and GPR data
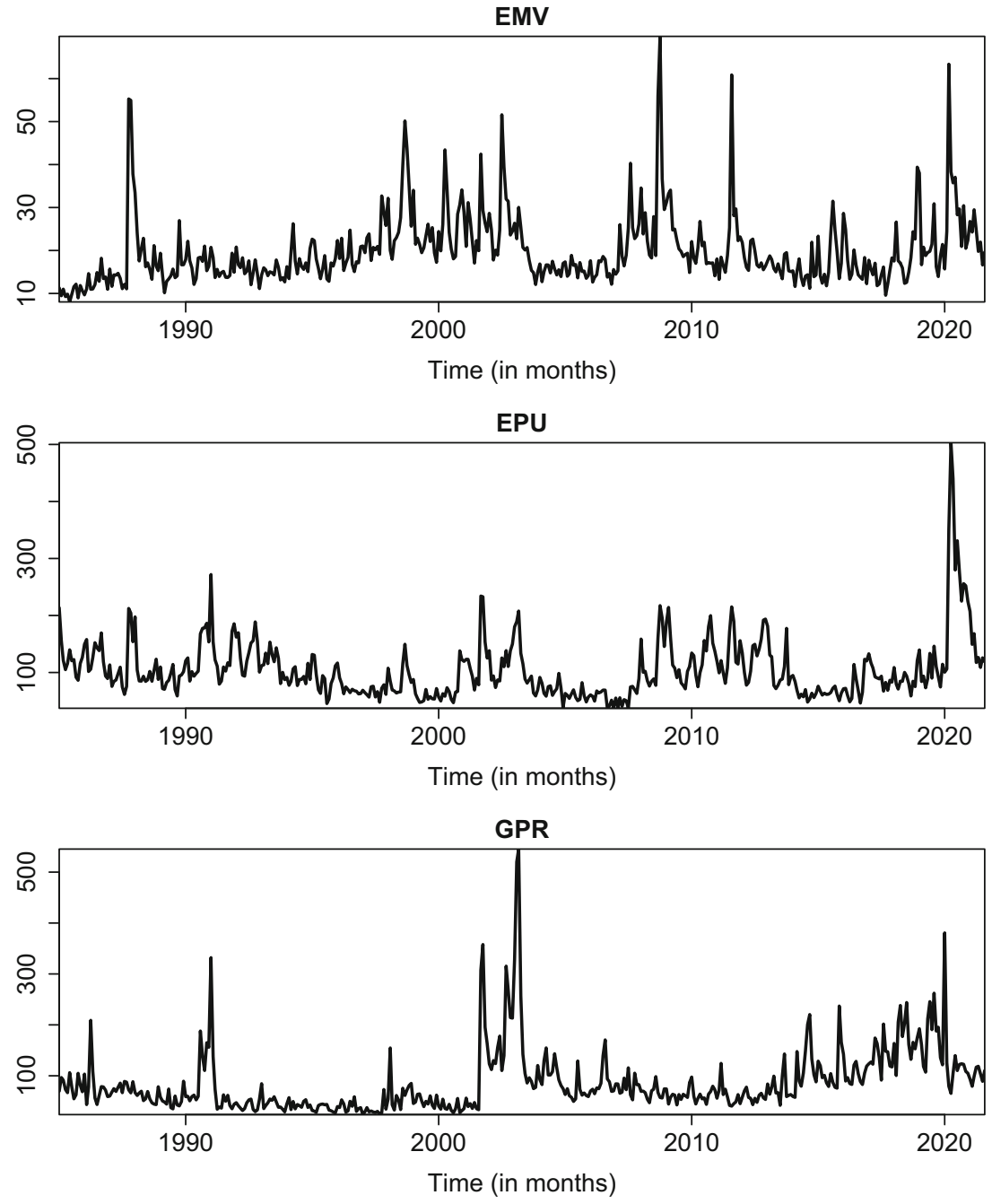

We estimate the function, $f$, that maps the predictors into the forecast of the subsequently realized variance by a machine-learning technique known as random-forest technique (Breiman 2001). A random forest consists of a large number of individual random regression trees and, thereby, belongs to the class of ensemble machine-learning techniques (see the textbook by Hastie et al. 2009). A regression tree consists of branches that recursively partition in a hierarchical and binary way the space of predictors into nonoverlapping regions. Growing a large regression tree and, thus, forming a large number of such regions makes it possible to compute increasingly granular predictions of the realized variance. At the same time, however, the complex hierarchical structure of a regression tree eventually results in an overfitting and data-sensitivity problem. A random forest addresses this problem by combining a large number of random individual regression trees. To this end, one draws a large number of bootstrap samples from the data and estimates a random regression tree on every bootstrap sample. A random regression tree differs from a standard regression tree in that a researcher uses a random subset of the predictors to grow the nodes and branches of the tree. This additional element of randomness mitigates the potential effect of influential individual predictors on tree building (which may result, for example, from outliers in the data). Moreover, because a random forest consists of a large number of random regression trees, it reduces the correlation of predictions computed by means of the individual random regression trees. Finally, upon computing the average of the predictions across the individual regression trees helps to stabilizes the random-forest-based forecasts of the realized variance of oil-price returns.

To sum up, for our forecasting experiment, we study four different models. For convenience, Table 1 provides summary information of the estimated models. Model 1 is the baseline AR model; Model 2 extends the baseline model to include the aggregate EMV, EPU, and GPR data; Model 3 uses the AR term along with the disaggregate EMV, EPU, 
Table 1 Estimated models

\begin{tabular}{lll}
\hline Model & Predictors & Estimator \\
\hline Model 1 & AR benchmark model & OLS \\
Model 2 & AR plus aggregate EMV, EPU, and GPR predictors & OLS \\
Model 3 & AR plus disaggregate EMV, EPU, and GPR predictors & Lasso \\
Model 4 & AR plus disaggregate EMV, EPU, and GPR predictors & Random forest \\
\hline
\end{tabular}

The Lasso estimator uses 10-fold cross-validation to determine the shrinkage parameter, where the optimal shrinkage parameter minimizes the mean cross-validated error. Random forests are built using 2000 individual random regression trees. Cross-validation is used to tune the number of predictors are chosen at random for tree building, the minimum number of data at a terminal node, and the parameter that controls the maximum imbalance of a split

and GPR data and is estimated by means of the Lasso; and Model 4 uses the same predictors as Model 3 but is estimated using random forests.

In our empirical analysis, we use the $\mathrm{R}$ language and environment for statistical computing (R Core Team 2021) to set up our forecasting experiment. We use the $\mathrm{R}$ addon package "glmnet" (Friedman et al. 2010) to estimate the Lasso model, where we use 10-fold cross-validation to determine the shrinkage parameter, where the optimal shrinkage parameter minimizes the mean cross-validated

Table 2 RMSFE ratios

\begin{tabular}{|c|c|c|c|c|c|c|}
\hline Models & Window & $h=1$ & $h=3$ & $h=6$ & $h=12$ & $h=24$ \\
\hline \multicolumn{7}{|c|}{ Recursive-estimation window } \\
\hline Model 1 vs. Model 2 & 60 & 0.9510 & 0.9672 & 0.9723 & 1.0083 & 1.0655 \\
\hline Model 1 vs. Model 2 & 120 & 0.9457 & 0.9641 & 0.9638 & 1.0085 & 1.0769 \\
\hline Model 1 vs. Model 3 & 60 & 1.0198 & 0.9566 & 0.9960 & 1.0648 & 1.2809 \\
\hline Model 1 vs. Model 3 & 120 & 0.9446 & 0.9539 & 0.8747 & 1.0623 & 1.3359 \\
\hline Model 2 vs. Model 3 & 60 & 1.0723 & 0.9890 & 1.0244 & 1.0560 & 1.2021 \\
\hline Model 2 vs. Model 3 & 120 & 0.9989 & 0.9894 & 0.9075 & 1.0534 & 1.2406 \\
\hline Model 1 vs. Model 4 & 60 & 1.0599 & 1.2019 & 1.1843 & 1.0883 & 1.4947 \\
\hline Model 1 vs. Model 4 & 120 & 0.9424 & 0.9417 & 0.9813 & 1.0950 & 1.5494 \\
\hline Model 2 vs. Model 4 & 60 & 1.1144 & 1.2427 & 1.2181 & 1.0793 & 1.4028 \\
\hline Model 2 vs. Model 4 & 120 & 0.9965 & 0.9767 & 1.0181 & 1.0857 & 1.4387 \\
\hline Model 3 vs. Model 4 & 60 & 1.0393 & 1.2564 & 1.1891 & 1.0221 & 1.1670 \\
\hline Model 3 vs. Model 4 & 120 & 0.9976 & 0.9872 & 1.1219 & 1.0307 & 1.1597 \\
\hline \multicolumn{7}{|c|}{ Rolling-estimation window } \\
\hline Model 1 vs. Model 2 & 60 & 0.9680 & 1.0117 & 0.9955 & 0.9898 & 1.1832 \\
\hline Model 1 vs. Model 2 & 120 & 0.8480 & 0.9611 & 0.9947 & 1.0684 & 1.2942 \\
\hline Model 1 vs. Model 3 & 60 & 1.0431 & 0.8969 & 1.0737 & 1.1060 & 1.3008 \\
\hline Model 1 vs. Model 3 & 120 & 0.9130 & 1.0464 & 1.0142 & 1.1407 & 1.4493 \\
\hline Model 2 vs. Model 3 & 60 & 1.0775 & 0.8866 & 1.0785 & 1.1174 & 1.0994 \\
\hline Model 2 vs. Model 3 & 120 & 1.0767 & 1.0887 & 1.0195 & 1.0677 & 1.1198 \\
\hline Model 1 vs. Model 4 & 60 & 1.0435 & 1.2044 & 1.1999 & 1.1474 & 1.5299 \\
\hline Model 1 vs. Model 4 & 120 & 0.8942 & 0.9634 & 1.0226 & 1.1492 & 1.7800 \\
\hline Model 2 vs. Model 4 & 60 & 1.0780 & 1.1905 & 1.2053 & 1.1593 & 1.2930 \\
\hline Model 2 vs. Model 4 & 120 & 1.0544 & 1.0023 & 1.0281 & 1.0757 & 1.3754 \\
\hline Model 3 vs. Model 4 & 60 & 1.0004 & 1.3429 & 1.1176 & 1.0375 & 1.1761 \\
\hline Model 3 vs. Model 4 & 120 & 0.9794 & 0.9207 & 1.0084 & 1.0075 & 1.2282 \\
\hline
\end{tabular}

The root-mean-square-forecast error (RMSFE) ratios are computed as the RMSFE of the forecast errors computed by means of the nominator model and the RMSFE of forecast errors computed by means of the denominator model. A RMSFE ratio larger than unity indicates that the denominator model produces more accurate forecasts in terms of the RMSFE criterion. The parameter $h$ denotes the forecast horizon (in months) 
error. We use the R add-on package "grf" (Tibshirani et al. 2021) to estimate random forests. Every random forest consists of 2000 individual random regression trees, and cross-validation is used to tune the tree parameters (number of randomly sampled predictors selected for tree building, minimum number of data at a terminal tree node, and maximum imbalance of a split at a node of a tree). For further details, we refer a reader to the documentation of these packages.

Because Fig. 1 clearly shows that the realized variances of oil returns exhibited large fluctuations during our sample period, we use recursive- and rolling-estimation windows to estimate our forecasting models. We analyze 5- and 10year windows (that is, windows of 60 and 120 months), where "window" in the case of the recursive-estimation window means the training period used to initialize the estimations.
In order to evaluate forecast accuracy, we use a wide array of standard statistics. First, we compute the rootmean-square-forecast-error (RMSFE) for every model, and then form the ratio of the RMSFE of two models to compare these two models. Second, we use the meanabsolute forecast error (MAFE) to compare the forecasts across models. The MAFE uses the L1 loss function to evaluate forecasts errors (the RMSFE uses the L2 loss function) and, thereby, is less sensitive to large forecast errors than the RMSFE. Occasional large forecast errors are likely to results from the peaks of the realized variance documented in Fig. 1. In order to assess whether our results are sensitive to large outliers, we also report results for $\sqrt{R V}$ when we use statistical tests to study forecast accuracy. Third, we use the Clark and West (2007) test to shed light on the statistical significance of differences of forecasts across models. The null hypothesis of the

Table 3 MAFE ratios

\begin{tabular}{|c|c|c|c|c|c|c|}
\hline Nominator vs. denominator model & Window & $h=1$ & $h=3$ & $h=6$ & $h=12$ & $h=24$ \\
\hline \multicolumn{7}{|l|}{ Recursive-estimation window } \\
\hline Model 1 vs. Model 2 & 60 & 0.9491 & 0.9799 & 0.9989 & 0.9923 & 1.0395 \\
\hline Model 1 vs. Model 2 & 120 & 0.9291 & 0.9687 & 0.9718 & 0.9718 & 1.0448 \\
\hline Model 1 vs. Model 3 & 60 & 0.9058 & 0.9014 & 0.9472 & 1.0298 & 1.2271 \\
\hline Model 1 vs. Model 3 & 120 & 0.8979 & 0.9184 & 0.8391 & 0.9598 & 1.2030 \\
\hline Model 2 vs. Model 3 & 60 & 0.9543 & 0.9199 & 0.9482 & 1.0378 & 1.1805 \\
\hline Model 2 vs. Model 3 & 120 & 0.9664 & 0.9480 & 0.8635 & 0.9876 & 1.1515 \\
\hline Model 1 vs. Model 4 & 60 & 0.9626 & 1.0280 & 1.0987 & 1.1788 & 1.4908 \\
\hline Model 1 vs. Model 4 & 120 & 0.9217 & 0.9542 & 0.9881 & 1.1600 & 1.5068 \\
\hline Model 2 vs. Model 4 & 60 & 1.0142 & 1.0491 & 1.0998 & 1.1880 & 1.4342 \\
\hline Model 2 vs. Model 4 & 120 & 0.9920 & 0.9850 & 1.0168 & 1.1937 & 1.4422 \\
\hline Model 3 vs. Model 4 & 60 & 1.0627 & 1.1404 & 1.1599 & 1.1447 & 1.2149 \\
\hline Model 3 vs. Model 4 & 120 & 1.0266 & 1.0390 & 1.1775 & 1.2087 & 1.2525 \\
\hline \multicolumn{7}{|l|}{ Rolling-estimation window } \\
\hline Model 1 vs. Model 2 & 60 & 0.9181 & 0.9753 & 0.9944 & 1.0148 & 1.2372 \\
\hline Model 1 vs. Model 2 & 120 & 0.7901 & 0.9442 & 0.9888 & 1.0643 & 1.2244 \\
\hline Model 1 vs. Model 3 & 60 & 0.9598 & 0.9871 & 1.1373 & 1.1272 & 1.4425 \\
\hline Model 1 vs. Model 3 & 120 & 0.8811 & 1.0104 & 0.9598 & 1.0796 & 1.3953 \\
\hline Model 2 vs. Model 3 & 60 & 1.0454 & 1.0121 & 1.1437 & 1.1107 & 1.1659 \\
\hline Model 2 vs. Model 3 & 120 & 1.1153 & 1.0702 & 0.9707 & 1.0144 & 1.1396 \\
\hline Model 1 vs. Model 4 & 60 & 0.9604 & 1.0385 & 1.1174 & 1.2505 & 1.6571 \\
\hline Model 1 vs. Model 4 & 120 & 0.8254 & 0.9444 & 1.0341 & 1.2474 & 1.7047 \\
\hline Model 2 vs. Model 4 & 60 & 1.0461 & 1.0648 & 1.1237 & 1.2322 & 1.3394 \\
\hline Model 2 vs. Model 4 & 120 & 1.0448 & 1.0002 & 1.0459 & 1.1721 & 1.3923 \\
\hline Model 3 vs. Model 4 & 60 & 1.0007 & 1.0521 & 0.9825 & 1.1094 & 1.1488 \\
\hline Model 3 vs. Model 4 & 120 & 0.9368 & 0.9346 & 1.0775 & 1.1555 & 1.2218 \\
\hline
\end{tabular}

The mean-absolute-forecast error $(M A F E)$ ratios are computed as the MAFE of the forecast errors computed by means of the nominator model and the MAFE of forecast errors computed by means of the denominator model. A MAFE ratio larger than unity indicates that the denominator model produces more accurate forecasts in terms of the MAFE criterion. The parameter $h$ denotes the forecast horizon (in months) 
Clark-West test is that a benchmark and a rival model produce the same mean-squared prediction error (MSPE). The alternative hypothesis is that the rival model produces a smaller MSPE than the benchmark model. Fourth, because Model 2 is not a strictly nested version of Models 3 and 4, and the linear Model 3 is not a strictly nested version of the nonlinear Model 4, we also compare (when we study a rolling-estimation window) the accuracy of forecasts by means of the Diebold and Mariano (1995) test, where we use the modified version of the test as suggested by Harvey et al. (1997). The null hypothesis is that of equal forecast accuracy across the benchmark and rival models. The alternative hypothesis is that the forecasts implied by the rival model are more accurate than the forecasts implied by the benchmark model. We shall report results for both the L1 and the L2 loss functions.

\section{Empirical results}

Table 2 summarizes root-mean-square-forecast-error (RMSFE) ratios. A ratio larger than unity shows that the benchmark model produces a larger RMSFE than the rival model. We report results for a recursive- and a rolling-estimation window, two window lengths, and five different forecast horizons. For the recursive-estimation window, we find that Model 2 produces worse forecasts then Model 1 for forecast horizons up to 6 months, and produces only moderately seized forecasting gains for a 12-month forecasting horizon. Similarly, Model 1 performs well when compared with Model 3, except for the two longest forecast horizons. The results further show that it is hardly possible to clearly rank Model 2 relative to Model 3. Model 3 outperforms Model 2 only for a forecast horizon of 1 and 2 years. In

Table 4 Baseline test results

\begin{tabular}{|c|c|c|c|c|c|c|}
\hline Benchmark vs. rival model & Window & $h=1$ & $h=3$ & $h=6$ & $h=12$ & $h=24$ \\
\hline \multicolumn{7}{|l|}{ Recursive-estimation window } \\
\hline Model 1 vs. Model 2 & 60 & 0.6935 & 0.5197 & 0.4857 & 0.4712 & 0.4391 \\
\hline Model 1 vs. Model 2 & 120 & 0.0842 & 0.0509 & 0.0882 & 0.3120 & 0.1444 \\
\hline Model 1 vs. Model 3 & 60 & 0.2231 & 0.2434 & 0.0203 & 0.3416 & 0.3062 \\
\hline Model 1 vs. Model 3 & 120 & 0.8563 & 0.1148 & 0.1174 & 0.2082 & 0.3789 \\
\hline Model 2 vs. Model 3 & 60 & 0.1169 & 0.0205 & 0.0074 & 0.2441 & 0.1246 \\
\hline Model 2 vs. Model 3 & 120 & 0.0199 & 0.0165 & 0.0497 & 0.4250 & 0.2008 \\
\hline Model 1 vs. Model 4 & 60 & 0.1346 & 0.1577 & 0.1075 & 0.0002 & 0.1427 \\
\hline Model 1 vs. Model 4 & 120 & 0.1541 & 0.1471 & 0.0337 & 0.0605 & 0.2544 \\
\hline Model 2 vs. Model 4 & 60 & 0.1100 & 0.1486 & 0.1011 & 0.0000 & 0.0365 \\
\hline Model 2 vs. Model 4 & 120 & 0.0019 & 0.0473 & 0.0275 & 0.0299 & 0.1326 \\
\hline Model 3 vs. Model 4 & 60 & 0.0084 & 0.1027 & 0.0101 & 0.0082 & 0.0002 \\
\hline Model 3 vs. Model 4 & 120 & 0.0004 & 0.0015 & 0.0011 & 0.0190 & 0.0039 \\
\hline \multicolumn{7}{|l|}{ Rolling-estimation window } \\
\hline Model 1 vs. Model 2 & 60 & 0.0469 & 0.0507 & 0.0077 & 0.0443 & 0.0068 \\
\hline Model 1 vs. Model 2 & 120 & 0.1346 & 0.0086 & 0.0053 & 0.0001 & 0.4706 \\
\hline Model 1 vs. Model 3 & 60 & 0.1360 & 0.4150 & 0.0012 & 0.1968 & 0.0160 \\
\hline Model 1 vs. Model 3 & 120 & 0.8476 & 0.0793 & 0.0531 & 0.0329 & 0.1973 \\
\hline Model 2 vs. Model 3 & 60 & 0.0513 & 0.4479 & 0.0002 & 0.1538 & 0.0003 \\
\hline Model 2 vs. Model 3 & 120 & 0.0072 & 0.0172 & 0.0253 & 0.1579 & 0.0110 \\
\hline Model 1 vs. Model 4 & 60 & 0.0893 & 0.1234 & 0.0676 & 0.0010 & 0.0214 \\
\hline Model 1 vs. Model 4 & 120 & 0.1340 & 0.0207 & 0.0008 & 0.0177 & 0.2152 \\
\hline Model 2 vs. Model 4 & 60 & 0.0372 & 0.1045 & 0.0466 & 0.0118 & 0.0024 \\
\hline Model 2 vs. Model 4 & 120 & 0.0069 & 0.0027 & 0.0017 & 0.0004 & 0.0068 \\
\hline Model 3 vs. Model 4 & 60 & 0.0012 & 0.1243 & 0.0995 & 0.0092 & 0.0173 \\
\hline Model 3 vs. Model 4 & 120 & 0.0632 & 0.0021 & 0.0005 & 0.0098 & 0.1504 \\
\hline
\end{tabular}

Results ( $p$-values; robust heteroskedasticity and autocorrelation consistent standard errors) of the Clark-West tests for an equal adjusted meansquared prediction error (MSPE). The alternative hypothesis is that the rival model produces a smaller MSPE than the benchmark model. The parameter $h$ denotes the forecast horizon (in months) 
sharp contrast, Model 4 produces more accurate forecasts in terms of the RMSFE statistic than the other three models for a training window of 60 months, and for the longer training window of 120 months when we consider the long forecast horizons. When we switch to a rolling-estimation window, we find again that Model 2 does not systematically perform better than Model 1. Model 3, in turn, produces better forecasts in terms of the RMSFE statistic than Models 1 and 2 for more combinations of rolling-estimation windows and forecast horizons than is the case for the recursive-estimation window, especially for the long forecast horizons. Model 4 outperforms the other three models for forecast horizons of $h \geq 6$, and for all forecast horizons when we consider the short training window.

In sum, we find that adding the aggregate EMV, EPU, and GPR data as predictors to the forecast model does not reliably improve forecast performance in terms of the RMSFE statistic relative to the simple benchmark AR model. Adding all disaggregated EMV, EPU, and GPR to the benchmark AR model and, given the large number of additional predictors, using the Lasso shrinkage estimator helps to improve forecast accuracy relative to the AR benchmark and the AR cum aggregate EMV and GPR predictors model, especially when we study a long forecast horizon and/or use a rolling-estimation window. Using the disaggregated data in conjunction with random forests, in turn, produces the best results relative to the other models at forecast horizons of $h \geq 6$, and also for the short forecast horizons when we opt for the short training/rolling window.

Because Fig. 1 witnesses that the realized variance of oilprice returns exhibited several sudden peaks during the sample period under scrutiny, we report in Table 3 the results we obtain when we focus on the mean-absolute forecast error (MAFE). The overall picture does not change. Model 4 systematically outperforms the other models except when we study one of the short forecast horizons and use a

Table 5 Test results $(\sqrt{R V})$

\begin{tabular}{|c|c|c|c|c|c|c|}
\hline Benchmark vs. rival model & Window & $h=1$ & $h=3$ & $h=6$ & $h=12$ & $h=24$ \\
\hline \multicolumn{7}{|l|}{ Recursive-estimation window } \\
\hline Model 1 vs. Model 2 & 60 & 0.9011 & 0.7817 & 0.2519 & 0.0006 & 0.0001 \\
\hline Model 1 vs. Model 2 & 120 & 0.8647 & 0.1791 & 0.0268 & 0.0005 & 0.0001 \\
\hline Model 1 vs. Model 3 & 60 & 0.9253 & 0.1127 & 0.0173 & 0.0004 & 0.0000 \\
\hline Model 1 vs. Model 3 & 120 & 0.2531 & 0.1241 & 0.0825 & 0.0109 & 0.0065 \\
\hline Model 2 vs. Model 3 & 60 & 0.1877 & 0.0622 & 0.0058 & 0.0002 & 0.0000 \\
\hline Model 2 vs. Model 3 & 120 & 0.0593 & 0.1132 & 0.0609 & 0.0105 & 0.0049 \\
\hline Model 1 vs. Model 4 & 60 & 0.1773 & 0.1831 & 0.0485 & 0.0000 & 0.0000 \\
\hline Model 1 vs. Model 4 & 120 & 0.4821 & 0.3011 & 0.0083 & 0.0091 & 0.0007 \\
\hline Model 2 vs. Model 4 & 60 & 0.1504 & 0.1613 & 0.0787 & 0.0000 & 0.0000 \\
\hline Model 2 vs. Model 4 & 120 & 0.3351 & 0.3923 & 0.0745 & 0.0007 & 0.0001 \\
\hline Model 3 vs. Model 4 & 60 & 0.1096 & 0.0151 & 0.0007 & 0.0005 & 0.0001 \\
\hline Model 3 vs. Model 4 & 120 & 0.1775 & 0.0081 & 0.0048 & 0.0022 & 0.0002 \\
\hline \multicolumn{7}{|l|}{ Rolling-estimation window } \\
\hline Model 1 vs. Model 2 & 60 & 0.7008 & 0.0984 & 0.0055 & 0.0005 & 0.0000 \\
\hline Model 1 vs. Model 2 & 120 & 0.2579 & 0.0111 & 0.0051 & 0.0007 & 0.0009 \\
\hline Model 1 vs. Model 3 & 60 & 0.5569 & 0.0526 & 0.0000 & 0.0001 & 0.0000 \\
\hline Model 1 vs. Model 3 & 120 & 0.7923 & 0.0260 & 0.0286 & 0.0010 & 0.0008 \\
\hline Model 2 vs. Model 3 & 60 & 0.0476 & 0.0131 & 0.0000 & 0.0000 & 0.0000 \\
\hline Model 2 vs. Model 3 & 120 & 0.2360 & 0.0077 & 0.0131 & 0.0001 & 0.0000 \\
\hline Model 1 vs. Model 4 & 60 & 0.1420 & 0.0737 & 0.0105 & 0.0000 & 0.0000 \\
\hline Model 1 vs. Model 4 & 120 & 0.4277 & 0.0072 & 0.0000 & 0.0000 & 0.0016 \\
\hline Model 2 vs. Model 4 & 60 & 0.0611 & 0.0710 & 0.0109 & 0.0177 & 0.0007 \\
\hline Model 2 vs. Model 4 & 120 & 0.2906 & 0.0721 & 0.0014 & 0.0000 & 0.0000 \\
\hline Model 3 vs. Model 4 & 60 & 0.0244 & 0.0862 & 0.0289 & 0.0015 & 0.0000 \\
\hline Model 3 vs. Model 4 & 120 & 0.0072 & 0.0024 & 0.0228 & 0.0010 & 0.0003 \\
\hline
\end{tabular}

Results ( $p$-values; robust heteroskedasticity and autocorrelation consistent standard errors) of the Clark-West tests for an equal adjusted meansquared prediction error (MSPE). The alternative hypothesis is that the rival model produces a smaller MSPE than the benchmark model. The parameter $h$ denotes the forecast horizon (in months) 
training window or rolling-estimation window of 10 years length. Model 2, in turn, has a hard time to beat Model 1 , the AR benchmark model, at the short forecast horizons and Model 3 only tends to produce better forecasts than Model 2 in terms of the MAFE statistic when we use a rolling-estimation window.

Next, we report in Table 4 the results of the Clark-West test for an equal mean-squared prediction error (MSPE). The alternative hypothesis is that the rival model produces a smaller MSPE than the benchmark model. Model 2 performs significantly better than Model 1 when we study a rolling-estimation window and when we consider a recursive-estimation window, but only when we combine a short forecast horizon with a long training period. Model 3, in turn, significantly outperforms Model 1 for several model configurations in case of the three long forecast horizons when we use a rolling-estimation window. Model 3 also significantly outperforms Model 2 mainly when we study an intermediate forecast horizon and a recursive-estimation window, and in seven out of ten model configurations when we analyze a rolling-estimation window. Hence, we find, on balance, evidence that using disaggregated EMV, EPU, and GPR data in conjunction with the Lasso estimator often helps improve forecast accuracy. Using random forests, however, even produces better forecasts in the majority of cases especially for the rolling-estimation window, where the overwhelming majority of test results for Model 4 is significant. For the recursive-estimation window, Model 4 performs better than Model 1 at the intermediate forecast horizons, and it performs better than Models 2 and 3 especially for the long forecast horizons and when we use the long training window.

Table 5 summarizes the test results that we obtain when we forecast the square-root of the realized variance of oil-price returns, that is, the realized volatility. For the recursive-estimation window, we find significant test results

Table 6 Diebold-Mariano test

\begin{tabular}{|c|c|c|c|c|c|c|}
\hline Benchmark vs. rival model & Window & $h=1$ & $h=3$ & $h=6$ & $h=12$ & $h=24$ \\
\hline \multicolumn{7}{|l|}{ Absolute-error loss } \\
\hline Model 1 vs. Model 2 & 60 & 0.9981 & 0.7268 & 0.5562 & 0.3889 & 0.0018 \\
\hline Model 1 vs. Model 2 & 120 & 1.0000 & 0.9142 & 0.5827 & 0.1205 & 0.0595 \\
\hline Model 1 vs. Model 3 & 60 & 0.8407 & 0.7056 & 0.0168 & 0.0512 & 0.0001 \\
\hline Model 1 vs. Model 3 & 120 & 1.0000 & 0.4309 & 0.6912 & 0.2090 & 0.0683 \\
\hline Model 2 vs. Model 3 & 60 & 0.1553 & 0.3832 & 0.0188 & 0.0639 & 0.0036 \\
\hline Model 2 vs. Model 3 & 120 & 0.0052 & 0.1137 & 0.6439 & 0.4137 & 0.1376 \\
\hline Model 1 vs. Model 4 & 60 & 0.8080 & 0.2727 & 0.0037 & 0.0000 & 0.0003 \\
\hline Model 1 vs. Model 4 & 120 & 1.0000 & 0.8600 & 0.2899 & 0.0001 & 0.0231 \\
\hline Model 2 vs. Model 4 & 60 & 0.1419 & 0.1734 & 0.0008 & 0.0001 & 0.0014 \\
\hline Model 2 vs. Model 4 & 120 & 0.1678 & 0.4979 & 0.1529 & 0.0000 & 0.0090 \\
\hline Model 3 vs. Model 4 & 60 & 0.4915 & 0.2155 & 0.6251 & 0.0478 & 0.0517 \\
\hline Model 3 vs. Model 4 & 120 & 0.9683 & 0.8788 & 0.1657 & 0.0115 & 0.0594 \\
\hline \multicolumn{7}{|l|}{ Squared-error loss } \\
\hline Model 1 vs. Model 2 & 60 & 0.9857 & 0.3872 & 0.5903 & 0.6343 & 0.0992 \\
\hline Model 1 vs. Model 2 & 120 & 0.9999 & 0.8701 & 0.5556 & 0.1435 & 0.1533 \\
\hline Model 1 vs. Model 3 & 60 & 0.2293 & 0.8752 & 0.1565 & 0.1646 & 0.0547 \\
\hline Model 1 vs. Model 3 & 120 & 0.9997 & 0.3337 & 0.4512 & 0.1813 & 0.1484 \\
\hline Model 2 vs. Model 3 & 60 & 0.0776 & 0.8334 & 0.1286 & 0.1124 & 0.0126 \\
\hline Model 2 vs. Model 3 & 120 & 0.0453 & 0.1580 & 0.4150 & 0.2222 & 0.1510 \\
\hline Model 1 vs. Model 4 & 60 & 0.2513 & 0.2109 & 0.0881 & 0.0077 & 0.0625 \\
\hline Model 1 vs. Model 4 & 120 & 0.9992 & 0.7599 & 0.3162 & 0.0650 & 0.1323 \\
\hline Model 2 vs. Model 4 & 60 & 0.0983 & 0.1984 & 0.0543 & 0.0065 & 0.0362 \\
\hline Model 2 vs. Model 4 & 120 & 0.1427 & 0.4750 & 0.1326 & 0.0088 & 0.1004 \\
\hline Model 3 vs. Model 4 & 60 & 0.4931 & 0.1800 & 0.2618 & 0.2986 & 0.0896 \\
\hline Model 3 vs. Model 4 & 120 & 0.7288 & 0.8762 & 0.4661 & 0.4541 & 0.0956 \\
\hline
\end{tabular}

Results ( $p$-values;) of the modified Diebold-Mariano test for equal accuracy of forecasts. Results are for a rolling-estimation window. The alternative hypothesis is that the rival model yields more accurate forecasts. The parameter $h$ denotes the forecast horizon (in months) 
in the vast majority of model configurations for forecast horizons of $h \geq 6$. Hence, Models 2 to 4 perform significantly better than the AR benchmark model, while Model 3 produces significantly better results than Model 2, and Model 4 performs better in a statistically significant way than the other three models. This is also true for a rolling-estimation window, where, in addition, several test results are significant also for a forecast horizon of three months. For a forecast horizon of 1 month, the majority of tests yields insignificant results, but Model 4 tends to perform better than Models 2 and 3 (but not Model 1) in a statistically significant way when we assume a rollingestimation window.

Finally, we present results for the modified DieboldMariano test in Table 6 for the case of a rolling-estimation window and an absolute-error loss and a squared-error loss function (at this stage, it should be noted that Models 1 to 3 are not strictly nested versions of Model 4 due to the nonlinear nature of the latter). According to the test results, the forecasts computed by means of Model 2 are significantly better than the forecasts computed by means of Model 1 only for the longest forecast horizon that we consider, $h=24$, and mainly for the absolute-error loss function. Moreover, the evidence that Model 3 yields systematically more accurate forecasts than Models 1 and 2 is concentrated, under the absolute-error loss function, at forecast horizons of $h \geq 6$. Finally, the accuracy of the forecasts extracted from Model 4 is significantly better than the accuracy of the forecasts implied by the other three models for the majority of model configurations for the two longest forecast horizons under consideration, $h=12,24$, for both loss functions (and for a few model configurations at $h=6)$.

\section{Concluding remarks}

Our findings, derived using machine-learning techniques, show that EMV, EPU, and GPR are useful for predicting the realized variance (and the realized volatility) of oilprice returns when the forecast horizon is sufficiently long. However, our findings also show that taking a disaggregated perspective by considering the various categories of EMV, EPU, and GPR may help to further improve the accuracy of forecasts of the realized variance of oil-price returns. This finding obtains (i) mainly at intermediate and long forecast horizons, and (ii) mainly when we use random forests to estimate our forecasting model. Hence, our findings suggest that a forecaster who needs forecasts at intermediate and long forecast horizons may find it useful to exploit the interplay of the disaggregated data and potential nonlinear links between the realized variance of oil-price returns and the disaggregated predictors when computing forecasts of the realized variance of oil-price returns. Besides this academic value, relatively more accurate volatility forecasts from disaggregated metrics of uncertainties compared to the aggregate counterparts of the same would assist oil-market participants in designing "optimal" investment portfolios in the wake of the recent financialization of the oil market. Finally, from a policy perspective, precise prediction of the future path of oil-market volatility by exploiting the informational content of various types of uncertainties using models of machine learning could aid policymakers to propose and implement fiscal and monetary policies ahead of time so as to prevent economic recessions, which are likely to follow increases in the variability in oil prices.

Our findings clearly show that it is interesting to explore whether a disaggregated view of uncertainty and geopolitical risks also is useful for forecasting the realized variances of the movements of the prices of other fossil resources and perhaps also those of agricultural commodities. In addition, the comparatively good performance of random forests in our forecasting experiment suggests that it is worthwhile to study the predictive performance of other widely studied machine-learning techniques like, for example, boosted regression trees for the realized variance of the returns of the oil price and other commodities. We leave this for future research.

Acknowledgements We would like to thank two anonymous referees for many helpful comments. However, any remaining errors are solely ours.

Author contribution Both authors contributed equally to the conception and design of the study. Material preparation and data collection was done by Rangan Gupta, and all empirical analyses were performed by Christian Pierdzioch. The first draft of the manuscript associated with the empirical segments of the paper, i.e., methodology and discussion of econometric results, was written by Christian Pierdzioch, while Rangan Gupta was involved in the writing of the introduction, literature, data, and conclusion sections of the paper. Both authors then read and approved the final manuscript.

Availability of data and materials The datasets used and/or analyzed in the current study are all publicly available as indicated in the data segment of the paper, and are also available from the corresponding author on a reasonable request.

\section{Declarations}

Ethics approval and consent to participate The authors mentioned in the manuscript have agreed for authorship, read and approved the manuscript, and given consent for submission and subsequent publication of the manuscript. The data used in this study does not involve human participants and/or animals.

Consent for publication Not applicable.

Conflict of interest The authors declare no competing interests. 


\section{References}

Andersen TG, Bollerslev T (1998) Answering the skeptics: yes, standard volatility models do provide accurate forecasts. Int Econ Rev 39(4):885-905

Asai M, Gupta R, McAleer M (2020) Forecasting volatility and covolatility of crude oil and gold futures: effects of leverage, jumps, spillovers, and geopolitical risks. Int J Forecast 36(3):933-948

Asai M, Gupta R, McAleer M (2019) The impact of jumps and leverage in forecasting the co-volatility of oil and gold futures. Energies 12:3379

Bakas D, Triantafyllou A (2018) The impact of uncertainty shocks on the volatility of commodity prices. J Int Money Financ 87:96-111

Bakas D, Triantafyllou A (2020) Commodity price volatility and the economic uncertainty of pandemics. Econ Lett 193:109283

Baker SR, Bloom NA, Davis SJ (2016) Measuring economic policy uncertainty. Q J Econ 131(4):1593-1636

Baker SR, Bloom NA, Davis SJ, Kost KJ (2019) Policy news and stock market volatility. NBER Working Paper No. 25720

Bernanke BS (1983) Irreversibility, uncertainty, and cyclical investment. Q J Econ 98(1):85-106

Bonaccolto M, Caporin M, Gupta R (2018) The dynamic impact of uncertainty in causing and forecasting the distribution of oil returns and risk? Physica A: Stat Mech Applic 507:446-469

Bonato M (2019) Realized correlations, betas and volatility spillover in the agricultural commodity market: what has changed? Journal of International Financial Markets, Institutions and Money 62:184202

Bonato M, Gkillas K, Gupta R, Pierdzioch C (2020) Investor happiness and predictability of the realized volatility of oil price. Sustainability 12:4309

Bouri E, Gkillas K, Gupta R, Pierdzioch C (2020) Infectious diseases, market uncertainty and realized volatility of oil. Energies 13(16):4090

Bouri E, Gupta R, Pierdzioch C, Salisu AA (2021) El Niño and forecastability of oil-price realized volatility. Theor Appl Climatol 144:1173-1180

Breiman L (2001) Random forests. Mach Learn 45:5-32

Caldara D, Iacoviello M (2019) Measuring geopolitical risk. Working Paper, Board of Governors of the Federal Reserve Board

Chan JC, Grant A (2016) Modeling energy price dynamics: GARCH versus stochastic volatility. Energy Econ 54:182-189

Clark TD, West KD (2007) Approximately normal tests for equal predictive accuracy in nested models. J Econ 138:291-311

Degiannakis S, Filis G (2017) Forecasting oil price realized volatility using information channels from other asset classes. J Int Money Financ 76:28-49

Demirer R, Gkillas K, Gupta R, Pierdzioch C (2021) Risk aversion and the predictability of crude oil market volatility. A forecasting experiment with random forests. Journal of the Operational Research Society. https://doi.org/10.1080/01605682.2021. 1936668

Demirer R, Gupta R, Ji Q, Tiwari AK (2019) Geopolitical risks and the predictability of regional oil returns and volatility. OPEC Energy Rev 43(3):342-361

Demirer R, Gupta R, Pierdzioch C, Shahzad SJH (2020) The predictive power of oil price shocks on realized volatility of oil: a note. Resour Polic 69(C): 101856

Demirer R, Gupta R, Suleman MT, Wohar ME (2018) Timevarying rare disaster risks, oil returns and volatility. Energy Econ 75(C):239-248

Diebold FX, Mariano RS (1995) Comparing predictive accuracy. J Business Econ Stat 13:253-263

Dutta A, Bouri E, Saeed T (2021) News-based equity market uncertainty and crude oil volatility. Energy 222:119930
Friedman J, Hastie T, Tibshirani R (2010) Regularization paths for Generalized Linear Models via coordinate descent. J Stat Softw 33(1):1-22

Gabauer D, Gupta R (2020) Spillovers across macroeconomic, financial and real estate uncertainties: a time-varying approach? Struct Chang Econ Dyn 52:167-173

Gkillas K, Gupta R, Pierdzioch C (2020) Forecasting realized oil-price volatility: the role of financial stress and asymmetric loss. J Int Money Financ 104(C):102137

Gupta R, Ma J, Risse M, Wohar ME (2018) Common business cycles and volatilities in U.S. states and MSAs: the role of economic uncertainty. J Macroecon 5:317-337

Gupta R, Pierdzioch C (2021) Forecasting the volatility of crude oil: the role of uncertainty and spillovers. Energies 14(14):4173

Harvey D, Leybourne S, Newbold P (1997) Testing the equality of prediction mean squared errors. Int J Forecast 13:281-291

Hastie T, Tibshirani R, Friedman J (2009) The elements of statistical learning: data mining, inference and prediction, 2nd edn. Springer, New York

Lee CC, Olasehinde-Williams G, Akadiri SS (2021) Are geopolitical threats powerful enough to predict global oil price volatility? Environ Sci Pollut Res 28:28720-28731

Li X, Wei Y, Chen X, Ma F, Liang C, Chen W (2020) Which uncertainty is powerful to forecast crude oil market volatility? New evidence. International Journal of Finance and Economics. https://doi.org/10.1002/ijfe.2371

Liang C, Wei Y, Li X, Zhang X, Zhang Y (2020) Uncertainty and crude oil market volatility: new evidence. Appl Econ 52(27):2945-2959

Liu J, Ma F, Tang Y, Zhang Y (2019) Geopolitical risk and oil volatility: a new insight. Energy Econ 84:104548

Luo J, Demirer R, Gupta R, Ji Q (Forthcoming) Forecasting oil and gold volatilities with sentiment indicators under structural breaks. Energy Economics

Lux T, Segnon M, Gupta R (2016) Forecasting crude oil price volatility and value-at-risk: evidence from historical and recent data. Energy Econ 56:117-133

Mei D, Ma F, Liao Y, Wang L (2020) Geopolitical risk uncertainty and oil future volatility: evidence from MIDAS models. Energy Econ $86: 104624$

Poon S-H, Granger CWJ (2003) Forecasting volatility in financial markets: a review. J Econ Lit 41(2):478-539

R Core Team (2021) R: a language and environment for statistical computing. R Foundation for Statistical Computing, Vienna. https://www.R-project.org/

Salisu A. A., Gupta R., Olaniran A. (2021a) The effect of oil uncertainty shock on real GDP of 33 countries: a global VAR approach. Applied Economics Letters. https://doi.org/10.1080/13504851. 2021.1983134

Salisu AA, Gupta R, Bouri E, Ji Q (2021b) Mixed-frequency forecasting of crude oil volatility based on the information content of global economic conditions. Journal of Forecasting. https://doi.org/10.1002/for.2800

Tibshirani J, Athey S, Sverdrup E, Wager S (2021) grf: generalized Random Forests. R package version 2.0.2. https://CRAN. $\mathrm{R}$-project.org/package $=$ grf

Tibshirani R (1996) Regression shrinkage and selection via the lasso. J R Stat Soc Ser B 58:267-288

Van Robays I (2016) Macroeconomic uncertainty and oil price volatility. Oxf Bull Econ Stat 78(5):671-693

van Eyden R, Difeto M, Gupta R, Wohar ME (2019) Oil price volatility and economic growth: evidence from advanced economies using more than a century of data. Appl Energy 233:612-621

Publisher's note Springer Nature remains neutral with regard to jurisdictional claims in published maps and institutional affiliations. 